SU-4240-532

UR-1306

ER-40685-755

March,1993

\title{
HEAVY QUARK SOLITONS
}

\author{
Kumar S. Gupta \\ University of Rochester, \\ Department of Physics and Astronomy, \\ Rochester, NY 14627 \\ and \\ M.Arshad Momen, J.Schechter and A. Subbaraman \\ Department of Physics, \\ Syracuse University \\ Syracuse, NY 13244-1130
}

\begin{abstract}
We investigate the heavy baryons which arise as solitonic excitations in a "heavy meson" chiral Lagrangian which includes the light vector particles. It is found that the effect of the light vectors may be substantial. We also present a simple derivation which clearly shows the connection to the CallanKlebanov approach.
\end{abstract}




\section{Introduction}

The problem of describing baryons containing heavy quarks $(c, b, \ldots)$ as Skyrme type solitons may be considered as an extension of the problem of describing strange "light" baryons. An approximation in which the strange quark is considered very heavy was actually discussed some time ago by Callan and Klebanov ${ }^{1}$ and others $^{2}$. In this picture one imagines a stationary $K$-meson to which is bound a nucleon treated as a soliton of the $S U(2) \times S U(2)$ effective chiral Lagrangian. Of course it is not clear that the approximation of treating the strange quark as "heavy" is the best one; comparable results may be obtained in other treatments ${ }^{3,4,5}$ which consider all the baryon octet members on the same footing. On the other hand, it is reasonable to expect that the bound state approach is a suitable one for the baryons containing $c$ and $b$ quarks.

An application to the heavy quark baryons based on a fairly literal extension of refs. $1-2$ has in fact been given ${ }^{6}$. More recently it has been realized that to go to the heavy quark limit one should do more than just let the heavy mass get large. One should ${ }^{7}$ at the same time keep the heavy particle four velocity fixed and impose the Isgur-Wise heavy spin symmetry. This has the consequence that a heavy vector particle should always appear together in a multiplet with the heavy pseudoscalar. The latter feature was not taken into account in refs.1 - 2, 6 and so the problem was recently reexamined in some papers by a San Diego-Caltech group ${ }^{8,9}$ and by a Hanyang-Seoul-Jagellonian-Saclay-Stony Brook group ${ }^{10,11}$.

In the present note we shall consider the problem when the light vectors are included in the chiral Lagrangian. It is known that this makes both the mesonic and solitonic sector more realistic for the light particles. It is also likely to be important for the interaction of the heavy particles with the light ones; for example, the semileptonic $D \rightarrow K^{*}$ transition appears to dominate over $D \rightarrow K \pi$.

In addition, we will give a method of calculation which appears a bit simpler than the previous ones and which makes clearer the connection with

ref. 1. This may be of relevance since there is apparently some disagreement between the two groups mentioned above. 


\section{Effective Lagrangian}

The light part of the effective Lagrangian of pseudoscalars and vectors, $\mathcal{L}_{\text {light }}$ which we shall employ has been discussed in a number of places; see ref. 12 for details and ref. 13 for an updating of the symmetry breaking part as well as further references. The relevant light fields belong to the $3 \times 3$ matrix of pseudoscalars, $\phi$ and to the $3 \times 3$ matrix of vectors, $\rho_{\mu}$. It is convenient to define objects which transform simply under the chiral group:

$$
\begin{gathered}
\xi=\exp \left(i \phi / F_{\pi}\right), \quad U=\xi^{2} \\
A_{\mu}^{L}=\xi \rho_{\mu} \xi^{\dagger}+\frac{i}{\tilde{g}} \xi \partial_{\mu} \xi^{\dagger}, \quad A_{\mu}^{R}=\xi^{\dagger} \rho_{\mu} \xi+\frac{i}{\tilde{g}} \xi^{\dagger} \partial_{\mu} \xi \\
F_{\mu \nu}(\rho)=\partial_{\mu} \rho_{\nu}-\partial_{\nu} \rho_{\mu}-i \tilde{g}\left[\rho_{\mu}, \rho_{\nu}\right]
\end{gathered}
$$

where $F_{\pi} \approx 0.132 \mathrm{GeV}$ and $\tilde{g} \approx 3.93$ for a typical fit. We can do without the explicit form of $\mathcal{L}_{\text {light }}$ here; it is given in (2.6), (2.7) and (2.13) of ref.12 (wherein $g$ should be replaced by $\tilde{g}$ ). We also do not need the details of the $S U(3)$ symmetry breaking discussed in ref. 13. Actually we will restrict ourselves to two flavors of light quarks for simplicity.

The new feature associated with the interactions of the heavy meson fields involves the use of a field ${ }^{14}$ combining the heavy pseudoscalar, $P^{\prime}$ and heavy vector, $Q_{\mu}^{\prime}$ at fixed 4-velocity, $V_{\mu}$ :

$$
H=\frac{1-i \gamma_{\mu} V_{\mu}}{2}\left(i \gamma_{5} P^{\prime}+i \gamma_{\nu} Q_{\nu}^{\prime}\right), \quad \bar{H}=\gamma_{4} H^{\dagger} \gamma_{4}
$$

Note that $H$ is here considered to have dimension of mass. $H$ is a $4 \times 4$ matrix in the Dirac space and it also carries an unwritten light flavor index. The chiral interactions of $H$ with the light pseudoscalars were discussed in ref. 15. The extension to including light vectors was given in ref. 16, which notation we precisely follow here, and in ref. 17. The total light-heavy interaction terms we shall require $\operatorname{are}^{16}$ :

$$
\begin{gathered}
\frac{\mathcal{L}_{\text {heavy }}}{M}=i V_{\mu} \operatorname{Tr}\left[H\left(\partial_{\mu}-i \alpha \tilde{g} \rho_{\mu}-i(1-\alpha) v_{\mu}\right) \bar{H}\right]+i d \operatorname{Tr}\left[H \gamma_{\mu} \gamma_{5} p_{\mu} \bar{H}\right] \\
+\frac{i c}{m_{v}} \operatorname{Tr}\left[H \gamma_{\mu} \gamma_{\nu} F_{\mu \nu}(\rho) \bar{H}\right]
\end{gathered}
$$


where $m_{v} \approx 0.77 \mathrm{GeV}$ is the light vector mass and

$$
v_{\mu}, p_{\mu}=\frac{i}{2}\left(\xi \partial_{\mu} \xi^{\dagger} \pm \xi^{\dagger} \partial_{\mu} \xi\right)
$$

Furthermore $M$ is the heavy meson mass. $\alpha, c$ and $d$ are dimensionless coupling constants for the light-heavy interactions. The choice $\alpha=1$ corresponds to a natural notion of light vector meson dominance, which is interesting to test. As seems appropriate for an initial treatment, we do not include terms in (2.3) which are higher order in $1 / M$, contain more derivatives of the light fields or involve light flavor symmetry breaking.

For treating the light baryon as a soliton which gets bound to the heavy meson, we shall need information about the classical soliton solutions of $\mathcal{L}_{\text {light }}$. First note the $2 \times 2$ matrix decomposition of $\rho_{\mu}$ :

$$
\rho_{\mu}=\frac{1}{\sqrt{2}}\left(\omega_{\mu} \mathbf{1}+\tau^{a} \rho_{\mu}^{a}\right)
$$

Then we have (see e.g. ref. 12) the classical "profiles":

$$
\begin{gathered}
U_{c}=\exp (i \hat{\mathbf{x}} \cdot \tau F(r)), \quad \omega_{0 c}=\omega(r), \\
\rho_{i c}^{a}=\frac{1}{\sqrt{2} \tilde{g} r} \epsilon_{i k a} \hat{\mathbf{x}}_{k} G(r),
\end{gathered}
$$

and $\omega_{i c}=\rho_{0 c}^{a}=0$. The boundary conditions for a finite energy baryon number one solution are:

$$
\begin{gathered}
F(0)=-\pi, \quad G(0)=2, \quad \omega^{\prime}(0)=0, \\
F(\infty)=G(\infty)=\omega(\infty)=0 .
\end{gathered}
$$

Typically, the opposite sign is taken for $F(0)$ as a kind of convention but the sign choice above gives the correct sign for both axial vector current and vector current matrix elements ${ }^{18}$.

\section{Overview of the approach}

The first step in the bound state approach is to find the channel in which the potential between the heavy meson and the nucleon as soliton is maximally 
attractive. Of course in order to get a positive parity heavy baryon we should consider the orbital angular momentum $l$ to be unity. In ref. 1, the attraction is found in the state with $l=1$ and grand spin, $\mathbf{G}=\frac{1}{2}$. The grand spin is the sum of the isotopic spin of the heavy meson, $\mathbf{I}$ and the angular momentum, $\mathbf{J}$; it enters in the first place because the Skyrme soliton ansatz, given in (2.6), mixes the isospin and ordinary spaces. We would like to display a suitable wavefunction for the heavy meson, $\bar{H}$ in the $l=1, \mathrm{G}=\frac{1}{2}$ channel. First note from (2.2) that when we specialize the heavy meson to be at rest $\left(V_{i}=0\right)$, the $4 \times 4$ matrix $\bar{H}$ has non-vanishing elements only in the lower left $2 \times 2$ subblock. The first index of this submatrix represents the spin of the light degrees of freedom within the heavy meson while the second index represents the spin of the heavy quark. Furthermore there is the unwritten bivalent isospin index. Specializing to this subblock we then write the $\bar{H}$ wavefunction as:

$$
\bar{H}_{l h}^{b}=(\hat{\mathbf{x}} \cdot \boldsymbol{\tau})_{b d} \frac{\epsilon_{l d}}{\sqrt{2}} \frac{u(r)}{\sqrt{4 \pi M}}
$$

Here, the radial wave function, $u(r)$ is taken in the first approximation to be localized at the origin, $r^{2} u^{2}(r) \approx \delta(r)$. Note that the quantity $\hat{\mathbf{x}}$ represents the angular part of the space wave function and the first factor couples it to the isospin index, $b$ to give $\mathrm{G}=\frac{1}{2}$. In turn this is coupled to the light spin index, $l$ with the Clebsch-Gordan coefficient $\frac{1}{\sqrt{2}} \epsilon_{l d}$ to give $\mathrm{G}=0$. Finally, the heavy spin index, $h$ is left uncoupled (as appropriate to the heavy spin symmetry) to give the desired net result $\mathrm{G}=\frac{1}{2}$.

To see that the wavefunction (3.1) gives attraction we consider the matrix element of the "potential" obtained from (2.3):

$$
V=-i M d \int d^{3} x \operatorname{Tr}\left(H \gamma_{i} \gamma_{5} p_{i} \bar{H}\right)+\cdots
$$

where only the $d$ term has been shown for simplicity. Substituting in (3.1), $p_{i}$ from (2.4) and (2.6) as well as $\gamma_{i} \gamma_{5} \rightarrow-i \sigma_{i}$ yields

$$
V=\frac{d}{2} F^{\prime}(0) \epsilon_{s c}\left(\sigma_{i}\right)_{s l}\left\{(\hat{\mathbf{x}} \cdot \boldsymbol{\tau})_{c a}\left[-\frac{1}{2} \tau_{i}+\hat{x}_{i} \hat{\mathbf{x}} \cdot \boldsymbol{\tau}\right]_{a b}(\hat{\mathbf{x}} \cdot \boldsymbol{\tau})_{b d}\right\} \epsilon_{l d}+\cdots
$$

Noticing that the object within the curly brackets is simply $\frac{1}{2}\left(\tau_{i}\right)_{c d}$ we easily get

$$
V=-\frac{3 d}{2} F^{\prime}(0)+\cdots
$$


This provides an attractive force in the $l=1, \mathrm{G}=\frac{1}{2}$ channel since $F^{\prime}(0)>0$ and $d$ is also expected to be positive. We will give the contributions to $V$ from the first and third terms in (2.3) in the next section; they do not appear to change the attraction to repulsion. However if we were to replace the Clebsch-Gordan coefficient in (3.1) by $\delta_{l 1} \delta_{d 1}$, which corresponds to coupling everything but the heavy quark spin to $G=1$, we would find that the $-\frac{3}{2}$ factor in (3.4) is replaced by $+\frac{1}{2}$ and repulsion. This is in agreement with refs. $8-9$; in our approach the $\hat{\mathbf{x}} \cdot \boldsymbol{\tau}$ factor appears in a very natural way and also there is no need to introduce a collective rotation factor at the present stage.

The next step is to introduce collective coordinates by allowing those constant transformations which leave the Lagrangian invariant to depend on time. First consider the soliton "rotational" modes, ${ }^{19} A(t)$; these are defined from the transformations

$$
\begin{gathered}
U(\mathbf{x}, t)=A(t) U_{c}(\mathbf{x}) A^{-1}(t) ; \boldsymbol{\tau} \cdot \boldsymbol{\rho}_{i}(\mathbf{x}, t)=A(t) \boldsymbol{\tau} \cdot \boldsymbol{\rho}_{i c}(x) A^{-1}(t) ; \\
\bar{H}(\mathbf{x}, t)=A(t) \bar{H}_{\text {bound }},
\end{gathered}
$$

where $\bar{H}_{\text {bound }}$ is effectively the wavefunction in (3.1) and $A(t)$ acts on its isospace index. Defining the angular velocity, $\Omega$ by $A^{\dagger} \dot{A}=\frac{i}{2} \boldsymbol{\Omega} \cdot \boldsymbol{\tau}$ and substituting $(3.5)$ into $\int d^{3} x\left(\mathcal{L}_{\text {light }}+\mathcal{L}_{\text {heavy }}\right)$ gives an additional contribution to the Lagrangian of the general form ${ }^{1}$ :

$$
\delta L=\frac{1}{2} K \Omega^{2}-\chi \Omega \cdot \mathbf{G}^{H}
$$

in which $K$ and $\chi$ represent spatial integrals over the profiles in (2.6) while $\mathbf{G}^{H}$, to the accuracy of interest, is the expectation value of the heavy particle grand-spin, $\mathbf{G}^{H}=\langle\boldsymbol{\tau} / 2\rangle+\cdots$. Note that at the collective Lagrangian level, as discussed in ref. 1, the angular momentum of the heavy field is represented by its grand-spin while the heavy field isospin is transmuted to zero. From (3.6), with the soliton angular momentum, $J_{i}^{s}=\partial \delta L / \partial \Omega_{i}$, the rotational collective Hamiltonian is obtained as

$$
H_{\text {coll }}=\frac{1}{2 K}\left(\mathbf{J}^{s}+\chi \mathbf{G}^{H}\right)^{2} .
$$

The moment of inertia, $K$ is identified from the light soliton sector as $K=$ $\frac{3}{2}\left(m_{\Delta}-m_{N}\right)^{-1}$ in terms of the nucleon and $\Delta$ masses. Eqn (3.7) can be 
simplified by noting that the total angular momentum is given as $\mathbf{J}=\mathbf{J}^{s}+$ $\mathbf{G}^{H}$. Then we arrive at the heavy baryon mass formula ${ }^{1}$ :

$$
H_{\text {coll }}=\frac{1}{3}\left(m_{\Delta}-m_{N}\right)[(1-\chi) I(I+1)+\chi J(J+1)+\cdots],
$$

where the three dots stand for a term which does not split the heavy baryon masses. In deriving (3.8), use was made of the fact that the soliton carries the full isospin of the heavy baryon at the collective level so that $\mathbf{J}^{2}=\mathbf{I}^{2}$. We will see that $\mathcal{L}_{\text {heavy }}$ in $(2.3)$ leads to $\chi=0$. Thus we have the final results:

$$
m\left(\Sigma_{Q}^{*}\right)-m\left(\Sigma_{Q}\right)=0 ; m\left(\Sigma_{Q}\right)-m\left(\Lambda_{Q}\right)=\frac{2}{3}\left(m_{\Delta}-M_{N}\right),
$$

wherein the subscript $Q$ denotes the heavy baryon in which the $s$ quark has been replaced by the heavy quark $Q$. The equality $m\left(\Sigma_{Q}^{*}\right)=m\left(\Sigma_{Q}\right)$ is, of course, expected from the heavy quark spin symmetry.

Finally, we take into account the translational mode, $\mathbf{R}(t)$ for the soliton by now setting

$$
U(\mathbf{x}, t)=A(t) U_{c}(\mathbf{x}-\mathbf{R}(t)) A^{-1}(t),
$$

and similarly treating the light vector fields. Imagining the heavy meson at

rest with the soliton moving about it, we have a Schrödinger equation for the relative motion

$$
\left(-\frac{1}{2 m_{N}} \nabla_{R}^{2}+V(\mathbf{R})\right) u(\mathbf{R})=E u(\mathbf{R})
$$

wherein $V(\mathbf{R})$ is to be identified with (3.4). It will be expanded to quadratic order

$$
V(\mathbf{R})=V_{0}+\frac{1}{2} \kappa \mathbf{R}^{2} \cdots,
$$

following the notation of ref. 9 .

\section{Effects of light vector fields}

Here we give details of the calculation, including all three terms in $\mathcal{L}_{\text {heavy }}$, (2.3). The $\alpha$ term involves the profile $\omega(r)$ and the $c$ term involves the profile 
$G(r)$. The needed factors for the non zero contributions to the "potential", $V(r)$ are

$$
\begin{array}{r}
\rho_{0}=\frac{1}{\sqrt{2}} \omega(r), \quad p_{i}=\frac{\sin F}{2 r}\left(\tau_{i}-\hat{x}_{i} \hat{\mathbf{x}} \cdot \boldsymbol{\tau}\right)+\frac{1}{2} F^{\prime} \hat{x}_{i} \hat{\mathbf{x}} \cdot \boldsymbol{\tau}, \\
\gamma_{i} \gamma_{j} F_{i j}(\rho)=\frac{i}{\tilde{g} r}\left[-G^{\prime} \boldsymbol{\sigma} \cdot \boldsymbol{\tau}+\frac{1}{r}\left(r G^{\prime}+G(G-2)\right) \hat{\mathbf{x}} \cdot \boldsymbol{\sigma} \hat{\mathbf{x}} \cdot \boldsymbol{\tau}\right] .
\end{array}
$$

We expand the profiles around the origin as follows:

$$
\begin{gathered}
F(r)=-\pi+r F^{\prime}(0)+\frac{1}{6} r^{3} F^{\prime \prime \prime}(0)+\cdots, \\
\omega(r)=\omega(0)+\frac{1}{2} r^{2} \omega^{\prime \prime}(0)+\cdots, \\
G(r)=2+\frac{1}{2} r^{2} G^{\prime \prime}(0)+\frac{1}{24} r^{4} G^{\prime \prime \prime \prime}(0)+\cdots,
\end{gathered}
$$

where powers were deleted so that the underlying fields are analytic ${ }^{9}$ in the Cartesian coordinates. Substituting (4.1) and (4.2) into (2.3) and evaluating the resulting expression in the attractive channel corresponding to wavefunction (3.1) enables us to identify the parameters of the "potential" as

$$
\begin{gathered}
V_{0}=-\frac{3}{2} d F^{\prime}(0)+\frac{3 c}{m_{v} \tilde{g}} G^{\prime \prime}(0)-\frac{\alpha \tilde{g}}{\sqrt{2}} \omega(0), \\
\kappa=d\left(-\frac{5}{6} F^{\prime \prime \prime}(0)+\frac{1}{3} F^{\prime}(0)^{3}\right)+\frac{c}{m_{v} \tilde{g}}\left(\frac{5}{6} G^{\prime \prime \prime \prime}(0)+\frac{1}{2} G^{\prime \prime}(0)^{2}\right)-\frac{\alpha \tilde{g}}{\sqrt{2}} \omega^{\prime \prime}(0) .
\end{gathered}
$$

If we choose to evaluate $V(r)$ in the "repulsive" channel mentioned after (3.4), the $c$ and $d$ terms in both (4.3) and (4.4) would be multiplied by $-1 / 3$ while the $\alpha$ terms would remain unchanged.

It is of course necessary to know the derivatives of the $F(r), G(r)$ and $\omega(r)$ profiles which appear above. These are found by solving the coupled differential equations which arise from minimizing the static energy of $\mathcal{L}_{\text {light }}$ (see (6.6) of ref 12) subject to the boundary conditions (2.7) above. For the typical best fit to meson and light baryon masses these are uniquely characterized by the starting values ${ }^{20}$ :

$$
F^{\prime}(0)=0.795 G e V, \quad G^{\prime \prime}(0)=-0.390 G e V^{2}, \quad \omega(0)=-0.094 G e V .
$$


From (4.5) and the differential equations it is straightforward to deduce the next higher derivatives:

$$
F^{\prime \prime \prime}(0)=-0.12 G e V^{3}, \quad G^{\prime \prime \prime \prime}(0)=0.35 G e V^{4}, \quad \omega^{\prime \prime}(0)=0.016 G e V^{3} .
$$

Finally, let us justify the vanishing of the $\chi \boldsymbol{\Omega} \cdot\langle\boldsymbol{\tau}\rangle / 2$ term in (3.6) which was used to obtain the mass formulas in (3.9). This term might arise when (3.5) is substituted into (2.3). We note that a possible contribution from the $d$ term would involve a factor $p_{4}$ and hence a factor $\gamma_{4} \gamma_{5}$, which has no components in the $2 \times 2$ subblock for which $\bar{H} H$ is non-vanishing. Similarly the relevant factor for the $c$ term involves a factor $\gamma_{k} \gamma_{4}$, which also has a vanishing overlap with $\bar{H} H$. For the first term in (2.3) we may explicitly check that the operator $\boldsymbol{\tau}$ has zero expectation value in the wavefunction (3.1).

\section{Results and discussion}

To gauge the accuracy of the heavy quark symmetry we may observe that the vector-pseudoscalar mass differences, which should vanish in the $M \rightarrow \infty$ limit, are respectively about $400 \mathrm{MeV}, 145 \mathrm{MeV}$, and $45 \mathrm{MeV}$ for $K^{*}-$ $K, D^{*}-D$ and $B^{*}-B$. From this point of view, the s-quark should not be considered heavy while it seems quite reasonable to treat the $b$-quark in this manner.

First let us look at the mass splitting relations (3.9), which are independent of the fine details of the model. Unfortunately there is presently not enough heavy baryon data to check the formula $m\left(\Sigma_{Q}^{*}\right)-m\left(\Sigma_{Q}\right)=0$; it is not surprising that one gets $193 \mathrm{MeV}$ instead of 0 when one uses the hyperons formed with the $s$-quark. For the relation $m\left(\Sigma_{Q}\right)-m\left(\Lambda_{Q}\right) \approx 195 \mathrm{MeV}$, one finds $77 \mathrm{MeV}$ with the ordinary hyperons but $170 \mathrm{MeV}$ with the $c$-quark hyperons. The fair agreement in the latter case is encouraging. The results in the former case show that the Callan-Klebanov model should not be applied to the strange hyperons if one goes to the true heavy meson limit.

Additional physical quantities may be estimated with the help of the "potential" for the bound state problem; substituting (4.5) and (4.6) into (4.3) and (4.4) yields:

$$
V_{0}=-1.19 d-0.39 c+0.26 \alpha G e V
$$




$$
\kappa=0.27 d+0.12 c-0.04 \alpha G e V^{3},
$$

for the typical choice of light particle Lagrangian parameters. This, of course, involves the three light-heavy coupling coefficients $d, c$ and $\alpha$. The value of $d$ has been discussed in the literature ${ }^{21}$; bounds are obtained which agree with a simple estimate ${ }^{16}$ based on pole dominance of the $D \rightarrow K$ transition form factor:

$$
d=\beta \frac{F_{\pi}}{F_{D}} \approx 0.53
$$

where $\beta \approx 1.0$ and we have used ${ }^{22} F_{D} \approx 250 \mathrm{MeV}$. We shall adopt (5.2) for definiteness. In contrast we are just at the initial stage for determining the values of $c$ and $\alpha$; the present model may assist the direct approach from meson weak transition matrix elements in this regard.

At lowest order, $V_{0}$ is a measure of the energy which binds the heavy meson and soliton together as a heavy baryon. Allowing for a zero point energy $Z$ for quantum fluctuations, we write

$$
m\left(\Lambda_{Q}\right)-m_{N}-M=V_{0}+Z
$$

The left hand side is about $-780 \mathrm{MeV}$ if we identify $Q=b$. If we first neglect $Z$ and set $\alpha=1$ ("vector meson dominance"), we can get an idea of the value of $c$ by putting (5.1) into (5.3). This gives us $c \approx 1.0$, which seems quite reasonable in that it is expected to be of the same order of magnitude as $d$. Examining the individual terms in (5.1) we see that the pion $(d)$ term is dominant but the $\rho$ meson $(c)$ contribution is $60 \%$ as large and also attractive while the omega $(\alpha)$ contribution is $40 \%$ as large and repulsive. Of course there can be different fits but this seems to be the typical situation. It is clear that the vector mesons can be expected to play a non-trivial role.

Using the numbers given above we find the parameter $\kappa$, which measures the strength of the $R^{2}$ part of the collective mode potential in (3.12), to be about $0.22 \mathrm{GeV}^{3}$. With (3.12) we note that (3.11) is just the Schrödinger equation for a spherical harmonic oscillator with angular frequency $\left(\frac{\kappa}{m_{N}}\right)^{\frac{1}{2}}$ $\approx 0.5 \mathrm{GeV}$. As is well known this results in a zero point energy, $Z \approx 0.75 \mathrm{GeV}$. Such a value is not a small perturbation to the right hand side of (5.3) so one might expect the collective ansatz in (3.10) to be suspicious. It would be more reliable to include the full non-linearities of the soliton profiles as well as to include higher derivative interactions in the light-heavy Lagrangian. This 
is a future job, but we can at least mention the parameters which will fit (5.3)

to experiment while retaining $Z=\frac{3}{2}\left(\frac{\kappa}{m_{N}}\right)^{\frac{1}{2}}$. Keeping $\alpha=1$ and $d=0.53$ would result in $c=4.1$ and correspondingly $\kappa=0.6 \mathrm{GeV}^{3}$. Such a value of $c$ is on the large side. An alternative fit is obtained by choosing $c=1$ and taking $d=0.7$ (the experimental bound); then we find $\alpha=-2$ (which does not agree with our intuitive notion of light vector meson dominance) and correspondingly $\kappa=0.39$. The two kinds of fits give different values for $\kappa$. Since the orbital excitations of the spherical harmonic oscillator are equally spaced at intervals given by the angular frequency, we would expect the first orbitally excited $\Lambda_{b}$ to be about $0.80 \mathrm{GeV}$ higher for the first fit while about $0.63 \mathrm{GeV}$ higher for the second fit. Another possible future source of information about $\kappa$ is the Isgur-Wise function for the $\Lambda_{b} \rightarrow \Lambda_{c}$ weak transition form factor. It was shown in ref. 9 that the non-perturbative part of this form factor is simply given by the overlap of the initial and final state spherical harmonic oscillator wave functions:

$$
\eta_{0}\left(V \cdot V^{\prime}\right)=\exp \left[\left(m_{N}^{3} / \kappa\right)^{1 / 2}\left(V \cdot V^{\prime}+1\right)\right],
$$

where the strict $M \rightarrow \infty$ limit was taken for simplicity of writing.

We would like to thank Herbert Weigel for helpful discussions. This work was supported in part by the U.S. Department of Energy under the contract number DE-FG-02-85ER40231 and also by the grant number DEFG02-91ER40685.

\section{References}

1. C.G.Callan and I. Klebanov, Nucl. Phys. B262,365(1985); C.G. Callan, K. Hornbostel and I. Klebanov, Phys. Lett. B202 (1988).

2. J. Blaizot, M. Rho and N. Scoccola, Phys. Lett B 209 ,27(1988); N. Scoccola, H. Nadeau, M. Nowak and M. Rho, ibid.201, 425(1988);D. Kaplan and I. Klebanov, Nucl. Phys. B335,45(1990).

3. H.Yabu and K. Ando, Nucl. Phys. B301, 601(1988). 
4. H. Weigel, J. Schechter, N. W. Park and Ulf-G. Meissner, Phys. Rev. D42, 3177(1990).

5. N. W. Park and H. Weigel, Nucl. Phys. A541,453(1992).

6. M. Rho, D. O. Riska and N. Scoccola, Z. Phys. A341, 343(1992).

7. E. Eichten and F. Feinberg,Phys. Rev. D 23,2724(1981); M. B. Voloshin and M. A. Shifman, Sov. J. Nucl. Phys. 45,292,(1987); N. Isgur and M. B. Wise, Phys. Lett. B232, 113 (1989); H. Georgi, Phys. Lett. B 240, 447 (1990).

8. E. Jenkins, A. Manohar and M. B. Wise,Caltech preprint CALT-681783-REV (hep-ph/9205243);Z. Guralnik, M. Luke and A. V. Manohar, Nucl. Phys. B390,474(1993); E. Jenkins and A. Manohar,Phys. Lett. B294,173(1992).

9. E. Jenkins, A. Manohar and M. Wise, UCSD preprint UCSD/PTH 92-27 (hep-ph/9208248).

10. M. Rho, Talk at Workshop on "Baryons as Skyrme Solitons", Sept.2830, 1992; Siegen, Germany (hep-ph /9210268).

11. D.P. Min, Y. Oh, B. Y. Park and M. Rho, Seoul preprint SNUTP 9278 (hep-ph/9209275); H. K. Lee, M. A. Nowak, M. Rho and I. Zahed, Stony Brook preprint NTG-92-20( hep-ph/9301242).

12. P. Jain, R. Johnson, Ulf-G. Meissner, N. W. Park and J. Schechter, Phys. Rev. D37,3252,(1988).

13. J. Schechter, A. Subbaraman and H. Weigel, Syracuse-Tübingen preprint SU-4240-525 (hep-ph/9211239).

14. J.D. Bjorken, in La Thuile Rencontres , 1990 , p.583; M.Wise, Phys. Rev.D45, R2188(1992); F. Hussain , J. Körner and G. Thompson, Ann. Phys. 206,334,(1991) .

15. M. Wise, ref. 14 above; T. M. Yan, H.Y. Cheng, C. Y. Cheung, G. L. Lin, Y. C. Lin and H. L. Yu,Phys. Rev. D46, 1148(1992); G. Burdman and J. F. Donoghue, Phys. Lett. B 280, 280 (1992); P. Cho, Phys. Lett. B 285, 145 (1992). 
16. J. Schechter and A. Subbaraman, Syracuse preprint SU-4240-519 (hepph/9209256).

17. R. Casalbuoni, A. Deandrea, N. Di Bartolomeo ,R. Gatto, F. Feruglio and G. Nardulli, Phys. Lett. B292, 371(1992); Phys. Lett. B294, 371(1992);Phys. Lett. B299,139(1993); N. Kitazawa and T. Kurimoto, Osaka preprint OS-GE-27-92 (hep-ph/9302296).

18. See Appendix B of N. W. Park, J. Schechter and H. Weigel, Phys. Rev. D41, 2836(1990) and also Z. Guralnik et al, ref. 8 above.

19. G. Adkins, C. Nappi and E. Witten, Nucl. Phys. B228, 552(1983).

20. We are grateful to Herbert Weigel for supplying these. The numbers given correspond to the choice of parameters $\gamma_{2}=\gamma_{3},\left|\tilde{g}_{V V \phi}\right|=$ $1.9,|\tilde{h}|=0.4$, as discussed in ref. 5 above, where slightly different conventions from the present one were employed. Symmetry breaking terms were also included although their effect on the profiles is small.

21. P. Cho and H. Georgi, Phys. Lett. B296,408(1992); J. Amundsen et. al., ibid, 415(1992).

22. J. Amundsen, J. Rosner, M. Kelly, N. Horwitz and S. Stone, Enrico Fermi Institute preprint, EFI 92-31 (hep-ph/9207235). 